\title{
Fast Eddy Current Compensation by Feedback Linearization Neural Networks: Applications in Diffusion-Weighted Echo Planar Imaging
}

\author{
SAN-CHAO HWANG, ${ }^{1}$ CHING YAO, ${ }^{2}$ CHAO-HSIEN HSIEH, ${ }^{1}$ JYH-HORNG CHEN ${ }^{1}$ \\ ${ }^{1}$ Interdisciplinary MRI/MRS Lab, Department of Electrical Engineering, 1 Roosevelt Road, Room 304, National \\ Engineering, National Taiwan University, Taipei, Taiwan \\ ${ }^{2}$ Division of Medical Engineering Research, National Health Research Institutes, Zhunan, Taiwan
}

\begin{abstract}
This study describes the application of the method of feedback linearization neural networks, known from neural network computing, to the problem of gradient preemphasis. This approach of preemphasis adjustment does not require an iterative procedure between measurement and adjustment, therefore is essentially instantaneous in its execution. Based on our study, gradient compensation determined by our procedure effectively suppressed eddy current-induced geometric distortion and spatial shift of diffusion-weighted EPI images. (؟) 2006 Wiley Periodicals, Inc. Concepts Magn Reson Part B (Magn Reson Engineering) 29B: 1-8, 2006
\end{abstract}

KEY WORDS: DW-EPI; eddy current; feedback linearization neural networks; preemphasis adjustment

\section{INTRODUCTION}

Diffusion-weighted magnetic resonance imaging (DWI) sensitizes the MR images to the diffusive mobility of water and maps water diffusion in tissue by means of the apparent diffusion coefficient (ADC) (I). It can highlight the microstructural characteristics of biological tissues and serve as a useful imaging tool for clinical diagnosis and basic medical research (1). Recently, this imaging technique was extended to diffusion tensor imaging (DTI) and diffusion spectrum imaging (DSI) that are used to track fiber connectivity of white matter $(2,3)$. A large number of images with different magnitudes and directions of

Received 27 June 2005; revised 18 October 2005; accepted 23 October 2005

Correspondence to: Jyh-Horng Chen; E-mail address: chen@me.ee.ntu.edu.tw

Concepts in Magnetic Resonance Part B (Magnetic Resonance Engineering), Vol. 29B(1) 1-8 (2006)

Published online in Wiley InterScience (www.interscience.wiley. com). DOI 10.1002/cmr.b.20058

() 2006 Wiley Periodicals, Inc. the diffusion sensitizing gradients need be acquired to estimate the diffusion properties. For efficiency, these images are usually acquired using diffusion-weighted echo-planar imaging sequence (DW-EPI). The rapid switching of the gradient pulses of DW-EPI can generate eddy currents in conducting surfaces surrounding the gradient coils. Although generation of eddy currents is greatly decreased in an actively shielded gradient system, this can still occur, especially when using large and rapidly rising and falling diffusion sensitization gradient pulses. Eddy current effects are the major source of geometric distortions in DW-EPI, in the form of shearing, scaling, and translation of the images. These geometric distortions are predominantly along the phase-encoding direction because the bandwidth in the phase-encoding direction is far lower than it is in the readout-encoding direction (4). In addition, according to the shifting and scaling properties of 2D Fourier transforms, the imaging distortions of shearing, scaling, and translation are due, respectively, to the residual eddy-current-induced gradient field in the readout, phase, and slice encoding direction. To avoid substantial errors in the calculated 
diffusion properties, these geometric distortions must be suppressed or corrected.

Several kinds of methods have been proposed to minimize the problem of eddy current-induced geometric distortions using modification of diffusion sensitization pulse $(5,6)$, image postprocessing (4), and gradient preemphasis (7-9). Pulse modification methods are used to reduce or cancel the eddy currents induced by diffusion sensitizing gradient during the EPI readout period. Image postprocessing methods, such as the iterative cross-correction algorithm, are used to measure and correct the image distortions after image acquisition. However, the basic approach is to apply gradient preemphasis, preventing eddy currents from occurring in the first place.

In the preemphasis adjustment, there is a need to find the optimized parameters of the preemphasis system, which are the magnitudes and the time constants (long, middle, and short term) of three firstorder high-pass filters to compensate for the eddycurrent effects. This is achieved by measuring the eddy-current-corrupted gradient waveform and then optimizing the preemphasis parameters to minimize the corruption. In the part of measuring the eddycurrent-corrupted gradient waveform, several methods have been proposed. The most direct approach is the pickup coil method in which the magnetic flux through the pickup coil is transduced to the voltage signal, then recovered to the gradient waveform by an integrator (7). The other approaches are based on measuring the phase evolution of MR signal. Owing to that, a residual gradient field speeds MR signal dephasing, and the dephasing is in direct proportion to the magnitude of the residual gradient field. One of these approaches, the multiple free-induction decays (FIDs) method (8) is mainly used to estimate the magnitudes of the eddy current at various delay times after a test gradient. Another $k$-space trajectories approach is achieved by calculating the phase difference of two DW-EPI signals with and without DW gradients (9). The most accurate approach is the Fourier transforms method (10) that is used to obtain the actual gradient waveform by performing 1D-Fourier transforms of a set of MR signals encoded by additional gradient pulses before acquisition. When performing the preemphasis adjustments, the optimum condition is usually achieved with iterative processing between measurement and adjustment, fitting the time constants and the magnitude parameters from long, middle, and short terms, adjusting one parameter at a time, based on Levenberg-Marquardt least-squares fitting and singular value decomposition $(8,9)$.

This study describes a simple and practical method of preemphasis adjustment based on feedback linear- ization neural networks. It is based on the criteria that a transfer function maps the original gradient waveform to the eddy-current-corrupted gradient waveform and the function is not-serious nonlinear. Then one can use a preemphasis treatment to change the response of the gradient system from an unknown nonlinear function to a desired linear function. This method, known as feedback linearization, is necessary to design a feedback control law for the gradient system. In our study, the feedback control law is designed by neural networks because it can be used to approximate a transfer function from its input/output relation with arbitrary accuracy. The gradient waveform of the eddy current fields induced by the DW gradients is measured with a modified pickup coil method. Based on the input/output relation of the gradient system, the problem of preemphasis adjustment can be considered as a process of tracking control. Two neural networks (11-13) are used to construct a feedback-linearization control system where the control signal (optimized preemphasis gradient waveform) can be simulated from a reference signal (desired output). Using this feedback-linearization technique, the optimized preemphasis gradient waveform can be determined directly without iterative processing. A notable feature of the method is the absence of the assumption of a linear system. The optimized compensated waveform can be applied directly to the gradient amplifiers. It is merely a matter of expediency that the compensation is realized via analog high-pass filters already existing in the scanner system.

\section{THEORY}

The diagram of the gradient waveform measurement and variable definition are shown in Fig. 1. The fundamental assumption is that there is a function which maps the input gradient waveform to the output gradient waveform. The mapping includes implicitly the effects of eddy currents, gradient coil geometry, and gradient amplifier performance. According to the input/output relation, the problem of preemphasis adjustment can be considered as a control design problem: selecting an optimized input waveform $u_{p}(t)$ to make $y(t)$ be something desirable.

The form of a single input/single output nonlinear control system is:

$$
\begin{aligned}
& \mathbf{x}=d(\mathbf{x} ; u ; t) \\
& y=m(\mathbf{x} ; u ; t)
\end{aligned}
$$


The vector $\boldsymbol{x}$ describes the system state at some time $t$, the $u(t)$ is the control input, the function $d$ describes the system's dynamics, and the function $m$ describes the output measurements. If function $d$ is completely known and is controllable (i.e., given an initial state $\boldsymbol{x}_{\boldsymbol{o}}$, the arbitrary state $\boldsymbol{x}$ is achievable), the form of exact feedback linearization will be derived. Equation [1] can be transformed by change of coordinates to become a canonical state-space form such as:

$$
\dot{x}(t)=\left[\begin{array}{c}
\dot{x}_{1} \\
\dot{x}_{2} \\
\vdots \\
\dot{x}_{n}
\end{array}\right]=\left[\begin{array}{c}
x_{2} \\
x_{3} \\
\vdots \\
f[x(t)]+\dot{g}[x(t)] u(t)
\end{array}\right] .
$$

If the exact feedback linearization is not obtained directly, in a not-serious nonlinear system, it can be derived by approximate linearization $(11,12)$. However, in our case, the knowledge of the gradient system is based on measurement and it is unnecessary to model the gradient system response with an analytic form. The following process, based on Eq. [3], is applicable:

Let $y(t)=m(x ; u ; t)=x_{n+1}(t)$, then [3] becomes

$$
y(t)=f[\mathbf{x}(t)]+g[\mathbf{x}(t)] u(t)
$$

In Fig. 2, the functions $f$ and $g$ are approximated by two separate neural networks NN-f and NN-g. A virtual control trajectory $w(t)$ is always chosen as "desired output" following a low-pass filter, for example, with two poles in $\mathrm{z}=0.7$ (i.e., a Z-transfer

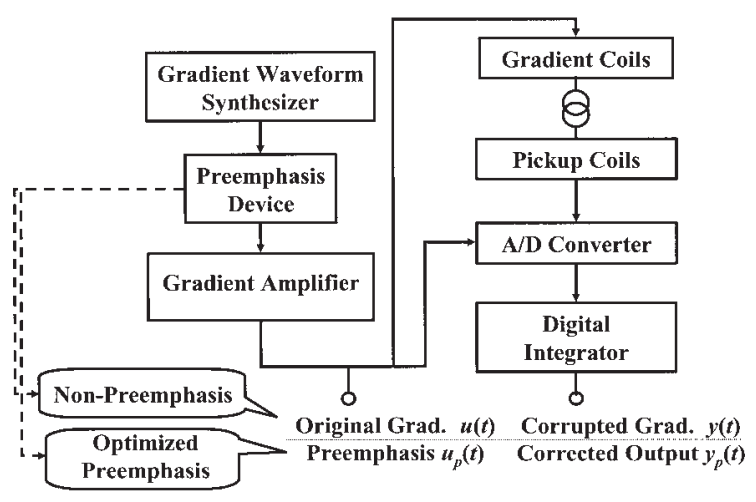

Figure 1 Diagram of the gradient waveform measurement, "Original Grad." $u(t)$ is the signal coming from current monitor of the gradient amplifier without preemphasis setting and "Corrupted Grad." $y(t)$ is the eddy-current-corrupted gradient waveform measured with pickup coils. The "Preemphasis" $u_{p}(t)$ is the signal coming from the current monitor of the gradient amplifier with optimized preemphasis setting and "Corrected Grad." $y_{p}(t)$ is the corresponding corrected gradient waveform.

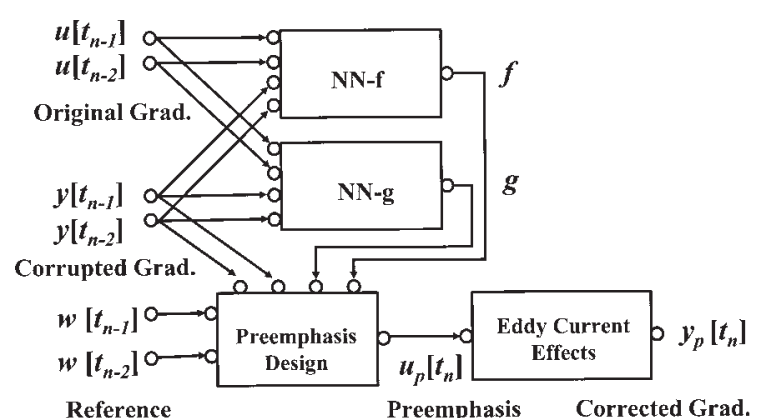

Figure 2 Diagram of feedback linearization neural networks, which are used to estimate the optimized preemphasis gradient waveform. The reference signal $w(t)$ is chosen as the original gradient waveform $u(t)$ treated with a lowpass filter (two poles in $\mathrm{z}=0.7$ ). Using input/output acquired data, the optimized preemphasis $u_{p}(t)$ can be solved by the method of feedback linearization neural networks.

function $\left.=1 /(z-0.7)^{2}\right)$ for smoothing the sharp turn. Using this $w(t)$ trajectory as the reference, the optimized preemphasis input $u_{p}(t)$ will be obtained by the following equation:

$$
u_{p}(t)=(w(t)-f[\mathbf{x}(t)]) / g[\mathbf{x}(t)]
$$

In practice, based on the following discrete model,

$$
\begin{gathered}
y(t)=f\left[y(t-1), \ldots, y\left(t-n_{a}\right), u\left(t-n_{k}-1\right)\right. \\
\left., \ldots, u\left(t-n_{k}-n_{b}\right)\right]+g[y(t-1), \ldots, \\
y\left(t-n_{a}\right), u\left(t-n_{k}-1\right), \ldots, \\
\left.u\left(t-n_{k}-n_{b}\right)\right] u\left(t-n_{k}\right),
\end{gathered}
$$

the optimized preemphasis input $u_{p}(t)$ can be solved by feeding the past signal $\left[y(t-1), \ldots, y\left(t-n_{a}\right)\right.$, $u\left(t-n_{k}-1\right), \ldots, u\left(t-n_{k}-n_{b}\right)$ and $\left.u\left(t-n_{k}\right)\right]$ to formula [6].

\section{MATERIAL AND METHODS}

This study contains three major procedures, including the gradient measurement, the preemphasis adjustment, and the imaging validation. First, the actual gradient waveform $y(t)$ and the signal coming from the "current monitor" of the gradient amplifier $u(t)$ are measured. Second, by the input/output acquired data, the optimized preemphasis gradient waveform $u_{p}(t)$ can be solved by the proposed method of feedback linearization neural networks. Finally, the phantom images of DW-EPI were performed to verify the setting of preemphasis adjustment. 


\section{Measurement of the Actual Gradient Field}

Experiments were performed on a $3 \mathrm{~T}$ whole-body system consisting of a Bruker Medspec 30/100 spectrometer (Bruker Medical, Ettlingen, Germany), an Oxford 3T-910AS (actively shield) magnet (Oxford Magnet Technology, Oxford, UK), a whole-body gradient coil (Bruker, BG630), and a birdcage resonator for head imaging.

The eddy current field induced by the DW gradient pulse in a standard DW-EPI sequence was measured with pickup coils. The pickup coils consist of six coils, namely the $x, y$, and $z$ pairs. These pairs contained a clockwise coil and an anticlockwise coil in series connection and placed at the opposite side of the gradient center for $x, y$, and $z$ (Fig. 3). This configuration was used to pick up the signal of the magnetic gradient field with eddy current effects to keep the spatial linear component of the eddy current field and cancel the spatially invariant component. The signal from pickup coils were captured with an AD/DA converter card in a personal computer (16 channels, 12-bit resolution, PCI 7012, Advantech) with $100 \mathrm{kHz}$ sampling rate. The captured data was then processed with digital integration as an actual gradient waveform "corrupted gradient" and compared with the signal coming from the current monitor of the gradient amplifier "original gradient." The signals of corrupted gradient and original gradient were acquired simultaneously with a synchronal trigger setting in the MRI sequence and the sequential acquisition delay of the multichannel signals of the AD/DA converter card was corrected. Moreover, an interactive user interface had been constructed to allow convenient control of processing and display of the multichannel data.

\section{Feedback Linearization Neural Networks}

With the acquired data (i.e., corrupted gradient and original gradient), two separate neural networks NN-f and NN-g were trained to approximate the functions $f$ and g. These were taken as two-layer neural networks with sigmoid functions in the hidden layer, and linear functions in the output layer. There were five nodes in the hidden layer and four nodes in the output layer. Theoretically, the combination of the nonlinear sigmoid function and the linear function is able to approximate an arbitrary function if there are enough nodes in these neural networks. However, the model of eddy-current effects is not so complicated, and a small number of nodes are found to be sufficient for approximating the functions $f$ and $g$. Levenberg-Marquardt's back propagation was used for the training
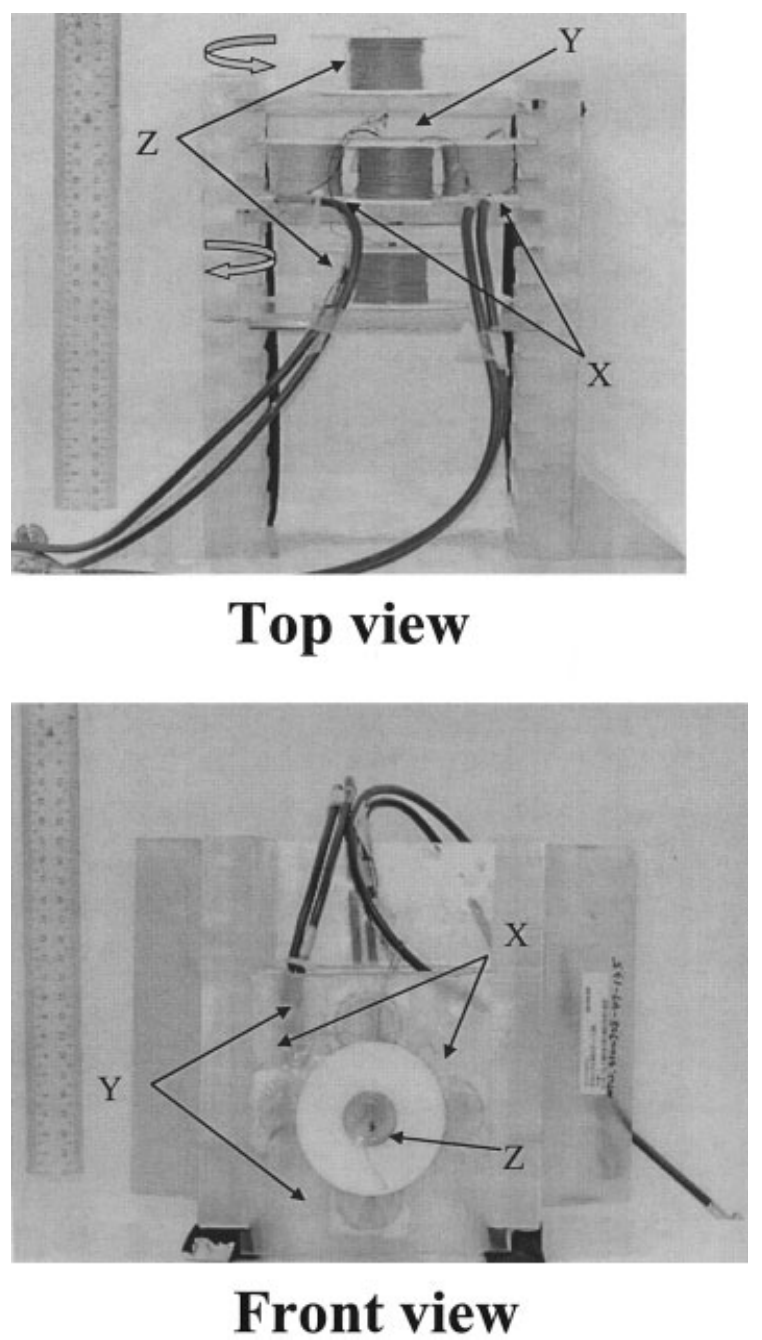

Figure 3 The configuration of the pickup coils of the $x, y$, and $z$ pairs. These pairs contained a clockwise coil and an anticlockwise coil in series connection and placed at the opposite side of the gradient center for $x, y$, and $z$. They keep the spatial linear component of the eddy-current-induced gradient field and cancel the spatially invariant component.

algorithm. After 100 epochs of training, about $7 \mathrm{sec}-$ onds computing time in a personal computer (Pentium $4,1.7 \mathrm{GHz}$ ), the mean squared errors of the estimation using these neural networks are less than $10^{-6}$. The reference $w(t)$ is chosen as the input $u(t)$ treated with a low-pass filter (two poles in $\mathrm{z}=0.7$ ). Following the processing of the feedback linearization neural networks described in the theory section, the optimized preemphasis $u_{p}(t)$ was obtained. Theoretically, $u_{p}(t)$ can be directly applied to the gradient amplifier, but usually the preemphasis is achieved with hardware approach by adding three first-order high-pass filter response of the applied gradient waveform itself. These six parameters (i.e., the magnitudes and the 
time constants of the three first-order high-pass filters) are used to synthesize the preemphasis waveform. The simplex algorithm (15) was used to determine these six parameters by minimizing the difference between the synthesized preemphasis waveform and the $u_{p}(t)$. It takes 10 seconds to perform the simplex algorithm in the personal computer. To validate the method of feedback linearization neural networks, a trapezoid function and its low-pass filter (one pole in $\mathrm{z}=0.9)$ response function, both with $1 \%$ random noise, was simulated.

\section{DW-EPI Acquisition}

Measurements and adjustments were performed for the DW gradient directions that were parallel to the Cartesian axes $x, y$, and $z$ of the gradient coil. The sequence parameters were as follows: $\mathrm{TE}_{\text {eff }}=44 \mathrm{~ms}$, DW gradient duration $\delta=30 \mathrm{~ms}$, evolution delay $\Delta=40 \mathrm{~ms}$, DW gradient strength $\mathrm{Gm}=10 \mathrm{mT} / \mathrm{m}$, yielding a $b$-value $B=194 \mathrm{~s} / \mathrm{mm}^{2}$. Asymmetric $k$ space coverage was performed along the phase-encoding direction (33\% of the data acquired prior to reaching the $k$-space center). EPI data matrix was $128 \times 128$, and sampling dwell time was $8.1 \mu \mathrm{s}$, which resulted in a duration of $132 \mathrm{~ms}$ for the EPI data collection. The experiment was multisliced into seven slices with a slice thickness of $8 \mathrm{~mm}$ and an interslice distance of $15 \mathrm{~mm}$. The optimized preemphasis adjustment was done by neural computation with the default $\mathrm{B}_{0}$ preemphasis adjustment done by the manufacturer.

\section{Measurement of the DW-EPI Geometric Distortion}

The cross-correlation method described by Haselgrove et al. was used to estimate the DW-EPI geometric distortions (4). In this method, the DW-EPI without the diffusion sensitizing gradients was set as the baseline image to analyze the geometric distortions along the phase-encoding orientation by applying the diffusion sensitizing gradients along the $x, y$, and $z$ orientations. The scaling factor is the only factor that needs to be optimized; once it has been determined, the other factors (i.e., shearing and translation) can be directly obtained by calculating. The scaling factor was selected from 0.9 to 1.1 with a 0.002 increased step, and it is determined under the criterion of the maximum correlation coefficient. Then the distortion images were multiplied with this optimized scaling factor and the displacements were estimated with the line-by-line cross-correlation again. Finally,

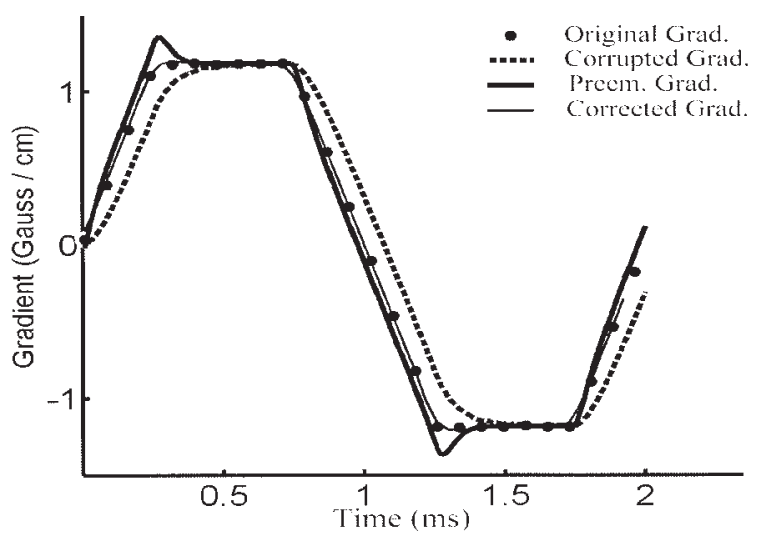

Figure 4 A set of simulated gradient waveforms, "Original Grad." $u(t)$, "Corrupted Grad." $y(t)$, "Preem Grad." $u_{p}(t)$, and "Corrected Grad." $y_{p}(t)$, were created to validate the accuracy of the feedback linearization neural networks. "Original Grad." is a trapezoidal function and its low-pass filter (one pole in $\mathrm{z}=0.9$ ) response function is defined as the "Corrupted Grad." One percent random noise was added to both. The preemphasis was calculated by the feedback linearization neural networks, and the "Corrected Grad." is the same low-pass filter (one pole in $\mathrm{z}=0.9$ ) response function of the preemphasis.

the linear regression of the displacements was performed to get the shearing and the translation factors.

\section{RESULTS}

The simulation (Fig. 4) shows the agreement between the original gradient and the corrected gradient waveform if there is adequate signal-to-noise ratio $(\mathrm{S} / \mathrm{N}>$ 100). In Fig. 5, the highly consistent result for a measured EPI readout trajectory waveform is similar to that of the simulation.

Shown in the windows with an interactive user interface (Fig. 6), the period in which the eddy-current-induced geometric distortions originate, is from the fall edge of the second diffusion pulse to the whole EPI acquisition windows. The gradient waveforms with nonpreemphasis and optimized preemphasis settings during the period of interest for the $x, y$, and $z$ orientations are shown in Fig. 7. The residual gradient fields are significantly reduced, and the actual gradient waveforms are tracking the input waveforms correctly. The error in tracking appears in the 0.3 -ms period behind the pulse edge because the input waveform has an apparent small distortion that the amplifier initially overshoots before setting to the correct value.

The measurements of the eddy-current-induced geometric distortions are shown in Fig. 8. In the case 


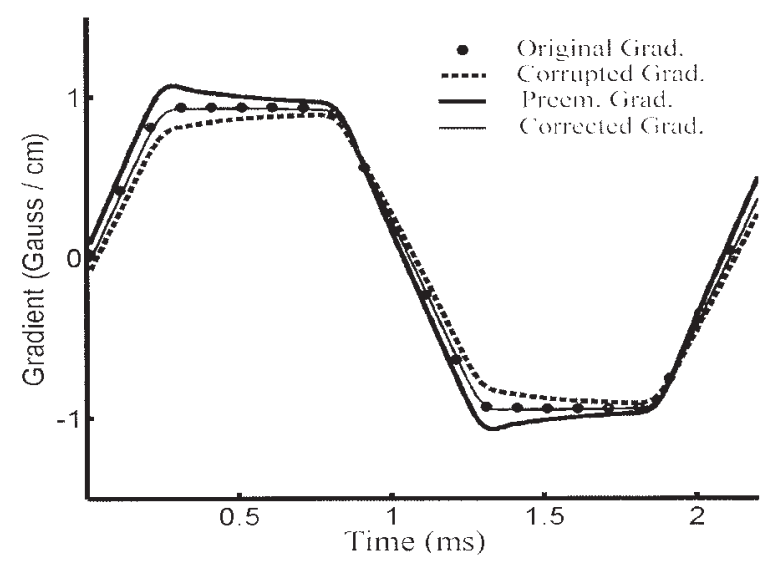

Figure 5 In the case of actual EPI gradient waveforms, the result of the feedback linearization neural networks method is presented. The "Original Grad." is coming from the "Current Monitor" of the gradient amplifier, which is without preemphasis. The "Corrupted Grad." is the measured gradient waveform. The "Preem. Grad." was calculated by the feedback linearization neural networks, and "Corrected Grad." is the measured gradient waveform. It shows high agreement between the original grad and the corrected grad, as in the simulation shown in Fig. 4.

of the nonpreemphasis, it shows the types of imaging distortion such as shearing, scaling, and translation. The existing residual gradient field along the readout orientation creates the image shearing, whereas the phase orientation creates the image scaling. The translation distortion is apparent in the existing slice orientation residual gradient field and in the diffusion gradient-induced $\mathrm{B}_{0}$ shift. After preemphasis adjustment, the distortions are reduced significantly (Fig. 9).

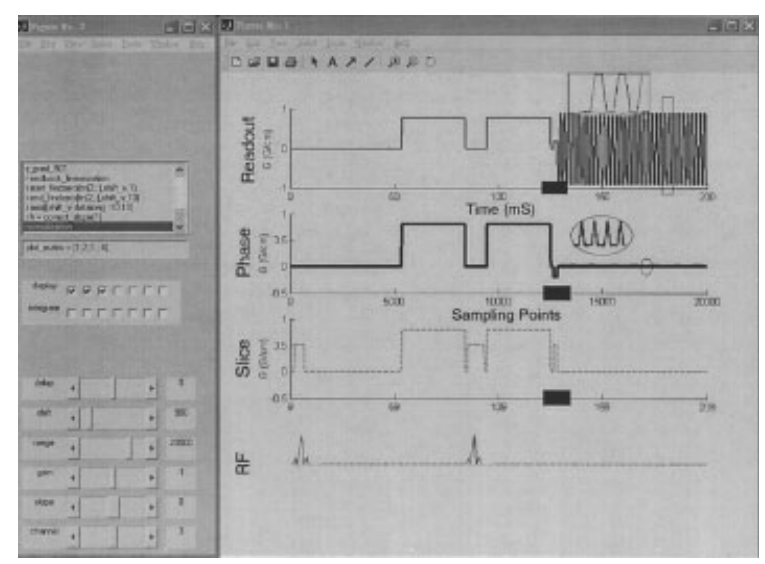

Figure 6 The user's interactive interface used to calculate and to show the data of multichannel gradient waveforms. The detail in the black-box region will be explored in Fig. 7.
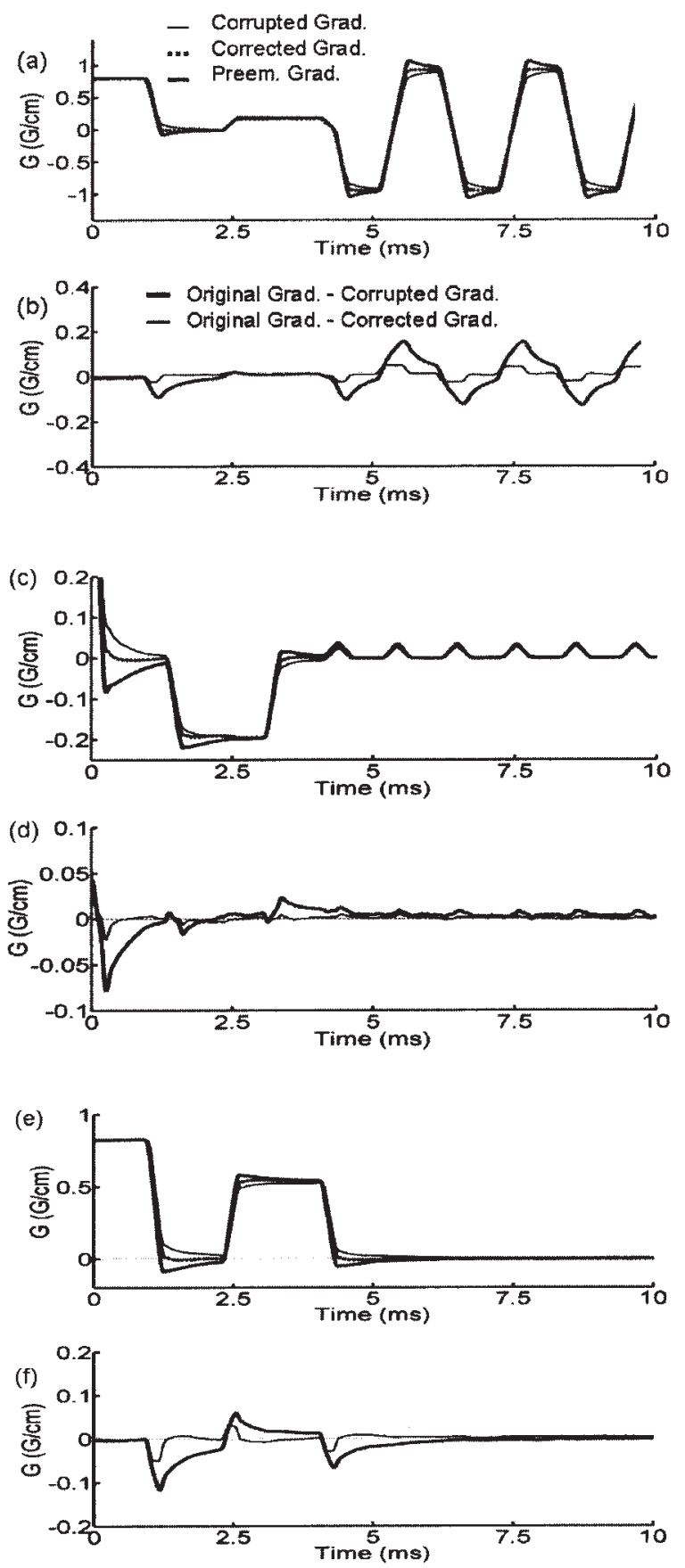

Figure 7 The portion of readout (a, b), phase (c, d), and slice (e, f) gradient waveform of DW-EPI (i.e., in the black-box region of Fig. 6) is shown. The plot of original gradient is skipped since it shows high agreement with the corrected gradient. In (b, d, and f), the errors are defined as the difference of original gradient and corrupted gradient (without preemphasis) and the difference of original gradient and corrected gradient (with optimized preemphasis). 

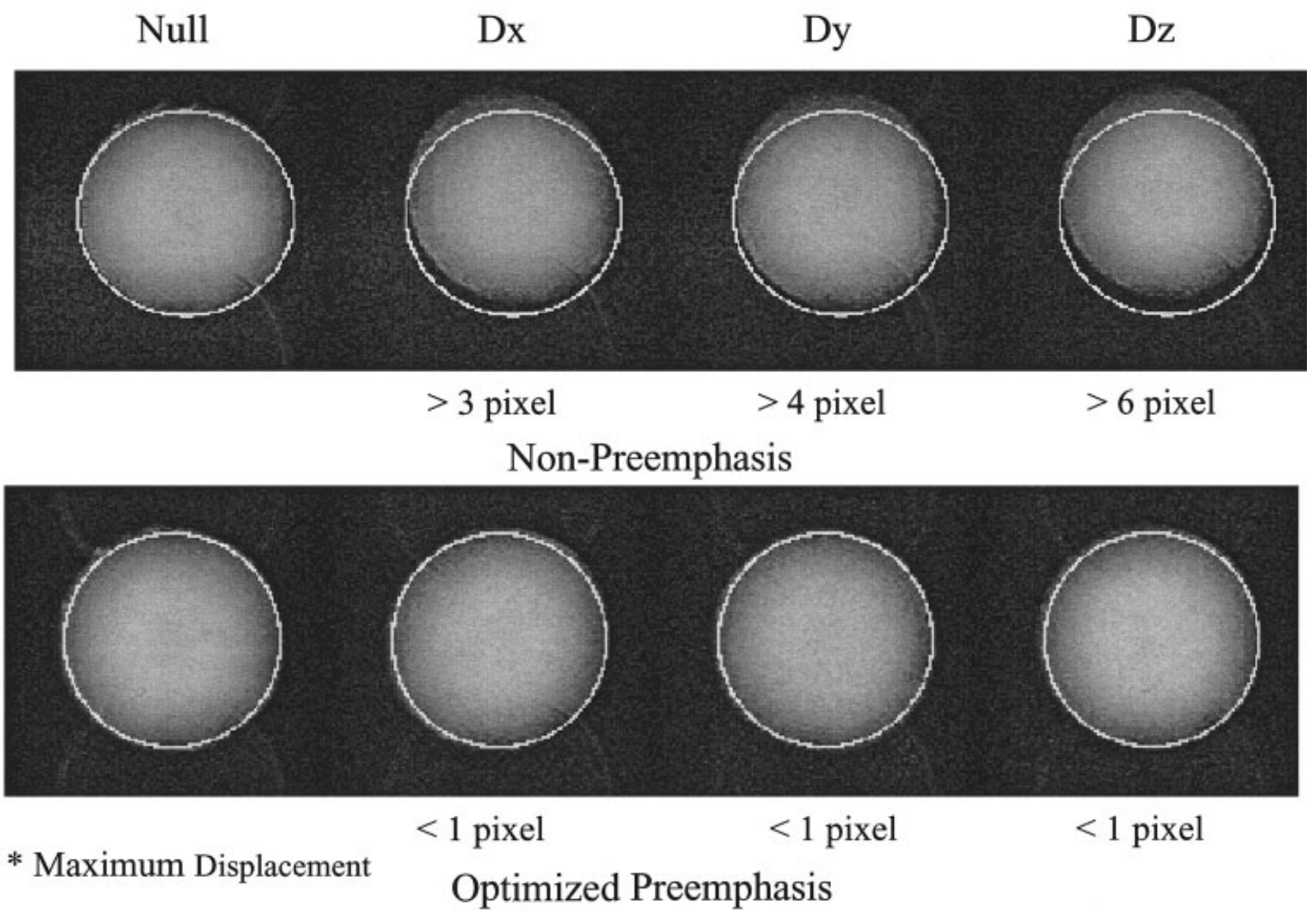

Figure 8 The DW-EPI images were acquired with nonpreemphasis and the conditions as below: without diffusion-sensitizing gradients (null); with diffusion-sensitizing gradients along $x, y$, and $z$ orientation (Dx, Dy, and Dz). The circles were derived from the baseline image (null) to enhance eddy-current-induced geometric distortions (upper row). Using the optimized preemphasis parameters, the maximum displacement of DW-EPI images are reduced from several pixels to less than one pixel (bottom row).

\section{DISCUSSION}

Gradient preemphasis adjustment is used to correct the imperfect gradient waveform from eddy-current effects. This is processed in two steps: first, to measure the gradient field and then to optimize the preemphasis parameters. Measuring the gradient field by the pickup coil method is direct and easy. It can explore the actual detail of a gradient pulse such as the eddy-current effects and the transient response of the gradient system. This information is important to design and tune the MRI sequence. This is the reason why we developed the interactive program to process and display the multichannel gradient waveforms. The advantages of the pickup coil method includes the following: not being limited to a specific duration in the acquisition windows of the MRI sequence; having a high temporal resolution; having the capability of simultaneous multichannel measurements; and elimi-

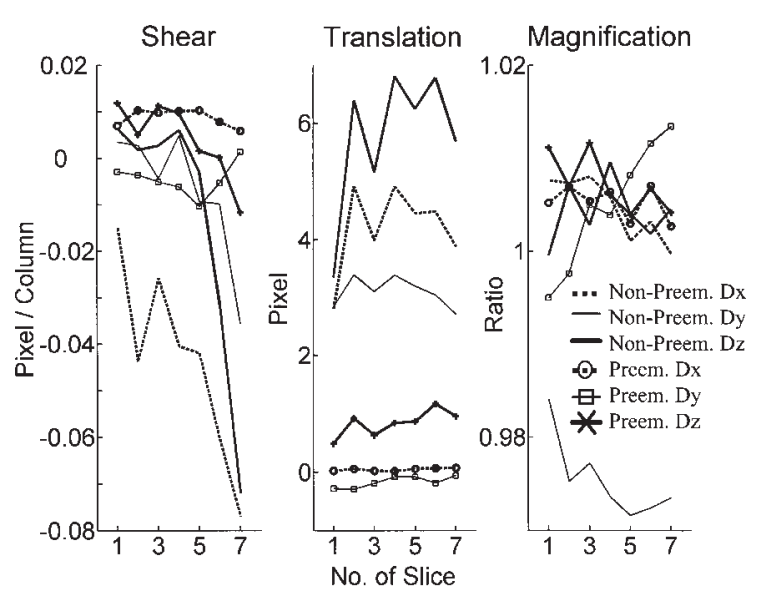

Figure 9 The geometric distortion of shearing, scaling, and translation from the nonpreemphasis setting (nonpreem) and optimized preemphasis setting (preem) are shown. The distortion evaluation was calculated from the images in Fig. 8 based on Haselgrove-Moore's cross-correlation method. 
nating the effects of the complicated MR acquisition conditions.

To optimize the preemphasis parameters, the processing of feedback linearization neural networks does not require a known system model but can be obtained by training the two neural networks and feeding in the past signal. This is easily accomplished in the case of simulation. However, in practical applications, such as the preemphasis adjustment, the implementation needs to eliminate the interruption of the input/output signals measurement. Because the geometric distortion of DW-EPI is highly sensitive to the residual eddy-current field, and the optimized preemphasis adjustment relies on the accuracy of gradient measurement, care must be taken with the following: maintaining the quality of the measured signal ( $\mathrm{S} / \mathrm{N}>100)$, calibrating the measurement equipment, calibrating the preemphasis hardware, and synchronizing the multichannel signals (the delay of the sequential acquisition of the ADC card).

Until now, the gradient field measurement with our pickup coils method concentrated on the spatially varying component of eddy-current-induced field. The $B_{0}$ preemphasis adjustment, which is used to correct the spatially invariant component of eddycurrent-induced field, will be performed in the future. Also, for verifying the pickup coil method, we will perform the gradient waveform measurement with the Fourier transform method in the future (10).

\section{CONCLUSION}

To find the optimized parameters to compensate for the eddy-current effects, most of the proposed preemphasis adjustment methods are achieved with an iterative procedure between measurement and adjustment. In our study, the preemphasis adjustment is achieved directly without iterative processing. Using this approach of feedback linearization neural networks, one can substantially reduce the eddy-currentinduced geometric distortions of the DW-EPI.

\section{ACKNOWLEDGMENT}

This work was supported by grants NSC92-2218-E-002018, NSC94-2752-H002-009-PAE, and 91-N-FA01-24-1 from the National Science Council, Taipei, Taiwan.

\section{REFERENCES}

1. Merboldt KD, Hanicke W, Frahm J. 1985. Self-diffusion NMR imaging using stimulated echoes. J Magn Reson 64:479-486.

2. Lin CP, Tseng WY, Cheng HC, Chen JH. 2001. Validation of diffusion tensor magnetic resonance axonal fiber imaging with registered manganese-enhanced tractography. Neuroimage 14:1035-1047.

3. Lin CP, Wedeen VJ, Chen JH, Yao C, Tseng WY. 2003. Validation of diffusion spectrum magnetic resonance imaging with manganese-enhanced rat optic tracts and ex vivo phantom. NeuroImage 19:1482-1495.

4. Haselgrove JC, Moore JR. 1996. Correction for distortions of echo-planar images used to calculate apparent diffusion coefficient. Magn Reson Med 36:960-964.

5. Alexander AL, Tsuruda JS, Parker DL. 1997. Elimination of eddy current artifacts in diffusion-weighted echo planar images: the use of bipolar gradients. Magn Reson Med 38:1016-1021.

6. Reese TG, Heid O, Weisskoff RM, Wedeen VJ. 2003. Reduction of eddy-current-induced distortion in diffusion MRI using a twice-refocused spin echo. Magn Reson Med 49:177-182.

7. Morich MA, Lampman DA, Dannels WR, Goldie FTD. 1988. Exact temporal eddy current compensation in magnetic resonance imaging systems. IEEE Trans Med Imaging 7:247-254.

8. Schmithorst VJ, Dardzinski BJ. 2002. Automatic gradient preemphasis adjustment: a 15-minute journey to improved diffusion-weighted echo-planar imaging. Magn Reson Med 47:208-212.

9. Papadakis NG, Martin KM, Pickard JD, Hall LD, Carpenter TA, Huang CL. 2000. Gradient preemphasis calibration in diffusion-weighted echo-planar imaging. Magn Reson Med 44:616-624.

10. Alley MT, Glover GH, Pelc NJ. 1998. Gradient characterization using a Fourier-transform technique. Magn Reson Med 39:581-587.

11. He S, Reif K, Unbehauen R. 1998. A neural approach for control of nonlinear systems with feedback linearization. IEEE Trans Neural Networks 9:1409-1421.

12. Nørgaard M. 2000. Neural network based system identification toolbox. Technical Report 00-E-891, Department of Automation, Technical University of Denmark.

13. Levin AU, Narendra KS. 1993. Control of nonlinear dynamical systems using neural networks: controllability and stabilization. IEEE Trans Neural Networks 4:192-206.

14. Nelder JA, Mead R. 1965. A simplex method for function minimization. Comp J 7:308-313. 